\title{
Practical Synthesis of 7-Prenylindole
}

\author{
Xin Xiong and Michael C. Pirrung ${ }^{\star}$ \\ Department of Chemistry, University of California, Riverside, CA 92521
}

\begin{abstract}
7-Prenylindole is a useful building block for natural product and natural product analog synthesis. While there have been several past syntheses of 7-prenylindole, none of them is very practical for its preparation on scale. Using an aza-Claisen rearrangement as the key step, 7-prenylindole has been prepared in four steps from indoline in $62 \%$ overall yield.
\end{abstract}

7-Prenylindole is a component of many natural products, such as the annonidines, ${ }^{1}$ astechrome (as its iron complex), ${ }^{2}$ and the asterriquinones ${ }^{3}$ (Chart 1 ). It is also a (simple) natural product, mostly from plants, in its own right. ${ }^{4}$ We have used 7-prenylindole in several syntheses of asterriquinone targets, in particular demethylasterriquinone $\mathrm{B} 1^{5}$ (a lead compound for oral insulin mimics), as have others. ${ }^{6}$ 7-Prenylindole has also served as a starting material for syntheses of the annonidines ${ }^{7}$ and astechrome. ${ }^{8}$ There are several literature preparations of 7 prenylindole, ${ }^{9}$ including one of our own. ${ }^{10}$ Because we identified the 7 -prenylindole region of demethylasterriquinone $\mathrm{B} 1$ as a major part of its pharmacophore, $5 \mathrm{c}$ we must prepare large numbers of compounds that include 7-prenylindole. This compound is therefore needed in ample supply, but in our experience all of its known preparations have drawbacks. Some do not scale up well, while others require expensive reagents or catalysts. We describe here a synthesis of 7-prenylindole that is readily performed with simple chemistry on a scale to prepare gram quantities.

The synthesis (Scheme 1) begins with indoline, which is $N$-dimethylpropargylated using classical methods ${ }^{11}$ to give the known compound $1 .{ }^{12}$ The semi-hydrogenation of compound 1 proceeds rapidly, so quickly in fact that prompt monitoring of the reaction, conducted merely with a balloon containing hydrogen, is required. Within 20-30 min, the known dimethylallyl amine $\mathbf{2}$ is produced. The acid-promoted aza-Claisen rearrangement of $N$-allylanilines ${ }^{13}$ is a known transformation. 9 a, 12 Heating 2 with trifluoroacetic acid in a microwave reactor introduces the prenyl group ortho to the nitrogen. Final oxidation of the indoline to the indole is conducted with manganese dioxide. ${ }^{14}$ Interestingly, when conducted in chloroform, this reaction gives appreciable quantities of compound $\mathbf{5}$. A putative mechanism to explain its formation is provided (Scheme 2), involving protonation (via $\mathrm{HCl}$ in the chloroform) of 7prenylindole by analogy to the observations of Van Vranken with tryptophan. ${ }^{15}$ Addition of 7-prenylindoline to the imminium ionresults in compound $\mathbf{4}$, which would presumably be more susceptible to $\mathrm{MnO}_{2}$ oxidation in the indolinering that is activated with two nitrogens. Unfortunately, this compound's structure is not related to any of the of dimeric 7-prenylindole natural products. As this reaction is a dimerization, it should be suppressed by conducting it at lower concentration, but given that significant quantities were obtained even at $0.1 \mathrm{M}$, further dilution was deemed impractical. Conducting the reaction in dichloromethane eliminates dimer formation. 
Each compound in Scheme 1 has been reported, as were each of the conversions shown. 16 Yet, none was described in sufficient detail to use this earlier work to prepare the target (in our hands). Overall, our synthesis proceeds in $62 \%$ yield. The fact that it gives the product in crystalline form when 7-prenylindole has such a low melting point attests to the purity of the material delivered by this synthetic route. It can be used to prepare gram batches of 7prenylindole in one run requiring approx. 3 days of laboratory time to execute. As such, this synthesis should greatly increase access to 7-prenylindole.

\section{EXPERIMENTAL}

\section{1-(2-methylbut-3-yn-2-yl)indoline (1)}

To a $50 \mathrm{~mL}$ round-bottomed flask containing indoline $(1.12 \mathrm{~mL}, 10.0 \mathrm{mmol})$, 2-chloro-2methylbut-3-yne (1.37 mL, $12.0 \mathrm{mmol})$, and $\mathrm{CuCl}(99 \mathrm{mg}, 1.0 \mathrm{mmol}$ ) inanhydrous THF (20 $\mathrm{mL})$ under an argon atmosphere was added $\mathrm{Et}_{3} \mathrm{~N}(1.67 \mathrm{~mL}, 12.0 \mathrm{mmol})$ dropwise at $0^{\circ} \mathrm{C}$. After the addition, the reaction mixture was stirred at room temperature overnight. The reaction mixturewas filtered through a plug of silica gel and concentrated under reduced pressure. The crude material waspurified by column chromatography $\left(\mathrm{CH}_{2} \mathrm{Cl}_{2}\right.$ :hexane 1:4) to give the title compound $\left(1.68 \mathrm{~g}, 91 \%\right.$ ) as a pale yellow oil. $\mathrm{R}_{\mathrm{f}}=0.34$ (ethyl acetate:hexane $1: 10$ ); IR (neat) 3286, 2985, 2844, 1605, 1483, 1254, 744 $\mathrm{cm}^{-1} ;{ }^{1} \mathrm{H}$ NMR $\left(300 \mathrm{MHz}, \mathrm{CDCl}_{3}\right) \delta 7.30(1 \mathrm{H}, \mathrm{d}$, $J=7.9 \mathrm{~Hz}), 7.187 .13(1 \mathrm{H}, \mathrm{m}), 6.80(1 \mathrm{H}, \mathrm{m}), 3.45(2 \mathrm{H}, \mathrm{t}, J=8.1 \mathrm{~Hz}), 2.98(2 \mathrm{H}, \mathrm{t}, J=8.1 \mathrm{~Hz})$, $2.46(1 \mathrm{H}, \mathrm{s}), 1.69(6 \mathrm{H}, \mathrm{s}) ;{ }^{13} \mathrm{C} \mathrm{NMR}\left(75 \mathrm{MHz}, \mathrm{CDCl}_{3}\right) \delta 150.2,131.8,127.0,124.6,118.6$, 111.9, 87.7, 71.1, 51.2, 49.7, 28.4, 27.4; HRMS Calcd. for $\mathrm{C}_{13} \mathrm{H}_{15} \mathrm{NH}^{+}(\mathrm{M}+\mathrm{H})^{+} 186.1278$, found 186.1278; Anal. Calcd. for $\mathrm{C}_{13} \mathrm{H}_{15} \mathrm{~N}$ : C, 84.28; H, 8.16; N, 7.56. Found: C, 84.21; H, $8.20 ; \mathrm{N}, 7.32$.

\section{1-(2-methylbut-3-en-2-yl)indoline (2)}

To a solution of alkyne $\mathbf{1}(3.18 \mathrm{~g}, 17.0 \mathrm{mmol})$ in $\mathrm{MeOH}(50 \mathrm{~mL})$ was added Lindlar catalyst (308 $\mathrm{mg}$ ). The reaction mixture was then degassed and stirred under an atmosphere of $\mathrm{H}_{2}$ (balloon with needle) until starting material disappears, within $30 \mathrm{~min}$, as indicated by TLC (longer exposure of the reactant to the reaction conditions leads to significant over-reduction, even complete conversion to the corresponding alkane). The reaction mixture was filtered through a short plug of silica gel and concentrated under reduced pressure. The crude material was purified by column chromatography (ethyl acetate:hexane 1:30) to yield the title compound $(2.73 \mathrm{~g}, 86 \%)$ as a colorless oil. $\mathrm{R}_{\mathrm{f}}=0.51$ (ethyl acetate:hexane 1:10); IR (neat) 2977, 2843, $1606,1483,1471,1458,1254,1195,913,743 \mathrm{~cm}^{-1} ;{ }^{1} \mathrm{H}$ NMR $\left(300 \mathrm{MHz}, \mathrm{CDCl}_{3}\right) \delta 7.07(1 \mathrm{H}$, d, $J=7.1 \mathrm{~Hz}), 6.96(1 \mathrm{H}, \mathrm{dt}, J=8.0,0.6 \mathrm{~Hz}), 6.80(1 \mathrm{H}, \mathrm{d}, J=8.0 \mathrm{~Hz}), 6.64(1 \mathrm{H}, \mathrm{m}), 6.15(1 \mathrm{H}$, $\mathrm{dd}, J=17.6,10.7 \mathrm{~Hz}), 5.24(1 \mathrm{H}, \mathrm{dd}, J=17.6,0.9 \mathrm{~Hz}), 5.15(1 \mathrm{H}, \mathrm{dd}, J=10.7,1.0 \mathrm{~Hz}), 3.44$ $(2 \mathrm{H}, \mathrm{t}, J=8.3 \mathrm{~Hz}), 2.92(2 \mathrm{H}, \mathrm{t}, J=8.3 \mathrm{~Hz}), 1.36(6 \mathrm{H}, \mathrm{s}) ;{ }^{13} \mathrm{C} \mathrm{NMR}\left(75 \mathrm{MHz}, \mathrm{CDCl}_{3}\right) \delta 151.0$, 147.3, 131.6, 126.8, 124.5, 117.5, 112.4, 111.4, 57.6, 49.3, 28.4, 24.4; HRMS Calcd. for $\mathrm{C}_{13} \mathrm{H}_{17} \mathrm{NH}^{+}(\mathrm{M}+\mathrm{H})^{+}$188.1435, found 188.1434; Anal. Calcd. for $\mathrm{C}_{13} \mathrm{H}_{17} \mathrm{~N}$ : C, 83.37; H, 9.15; N, 7.48. Found: C, 83.36; H, 9.20; N, 7.44.

\section{7-(3-methylbut-2enyl)indoline (3)}

To a solution of $2(1.87 \mathrm{~g}, 10.0 \mathrm{mmol})$ in toluene $(3 \mathrm{~mL})$ was added TFA $(0.1 \mathrm{~mL}, 1.3 \mathrm{mmol})$. The reaction mixture and a magnetic stir bar were sealed in the reaction vessel of a Discover ${ }^{\circledR}$ monomode microwave apparatus (CEM) and irradiated for $10 \mathrm{~min}$ at $150{ }^{\circ} \mathrm{C}$. The reaction mixture was concentrated in vacuo and purified by chromatography on silica gel (ethylacetate:hexane 1:8) to give the title compound $(1.69 \mathrm{~g}, 90 \%)$ as a pale yellow oil. $\mathrm{R}_{\mathrm{f}}=$ 0.20 (ethylacetate:hexane 1:10); IR (neat) 3375, 2969, 2913, 2850, 1601, 1455, 1306, 1254, $1059,748 \mathrm{~cm}^{-1} ;{ }^{1} \mathrm{H}$ NMR $\left(300 \mathrm{MHz}, \mathrm{CDCl}_{3}\right) \delta 7.02(1 \mathrm{H}, \mathrm{dd}, J=7.2,0.6 \mathrm{~Hz}), 6.88(1 \mathrm{H}, \mathrm{d}$, $J=7.5 \mathrm{~Hz}), 6.70(1 \mathrm{H}, \mathrm{t}, J=7.4 \mathrm{~Hz}), 5.27(1 \mathrm{H}, \mathrm{m}), 3.70(\mathrm{NH}, \mathrm{br} \mathrm{s}), 3.58(2 \mathrm{H}, \mathrm{t}, J=8.4 \mathrm{~Hz})$, $3.20(2 \mathrm{H}, \mathrm{d}, J=7.1 \mathrm{~Hz}), 3.06(2 \mathrm{H}, \mathrm{t}, J=8.4 \mathrm{~Hz}), 1.78-1.75(6 \mathrm{H}, \mathrm{m}) ;{ }^{13} \mathrm{C} \mathrm{NMR}(75 \mathrm{MHz}$, 
$\left.\mathrm{CDCl}_{3}\right) \delta 150.2,133.4,129.3,127.3,122.9,122.6,122.1,119.1,47.5,30.8,30.3,26.0,18.1$; HRMS Calcd. for $\mathrm{C}_{13} \mathrm{H}_{17} \mathrm{NH}^{+}(\mathrm{M}+\mathrm{H})^{+} 188.1435$, found 188.1436; Anal. Calcd. for $\mathrm{C}_{13} \mathrm{H}_{17} \mathrm{~N}$ : C, 83.37; H, 9.15; N, 7.48. Found: C, 83.34; H, 9.27; N, 7.42.

\section{7-(3-methylbut-2-enyl)-1H-indole}

A mixture of 3 ( $0.42 \mathrm{~g}, 2.2 \mathrm{mmol})$ and activated manganese(IV) oxide $(0.57 \mathrm{~g}, 6.6 \mathrm{mmol})$ in dichloromethane $(20 \mathrm{~mL})$ was heated at reflux. After $1.5 \mathrm{~h}$, additional activated manganese (IV) oxide $(0.57 \mathrm{~g}, 6.6 \mathrm{mmol})$ was added to the reaction mixture. The mixture was kept refluxing for another $1.5 \mathrm{~h}$, allowed to cool, and filtered through a short plug of silica gel. Concentration of the filtrate under reduced pressure was followed by column chromatography (ethyl acetate:hexane 1:30) to yield the title compound $(0.36 \mathrm{~g}, 88 \%)$ as an off-white solid, $\mathrm{mp}$ 43-44 ${ }^{\circ} \mathrm{C}$ (lit., $1 \mathrm{mp} 43-44{ }^{\circ} \mathrm{C}$ ). $\mathrm{R}_{\mathrm{f}}=0.21$ (ethyl acetate:hexane 1:10); ${ }^{1} \mathrm{H}$ NMR $(300 \mathrm{MHz}$, $\left.\mathrm{CDCl}_{3}\right) \delta 8.15(\mathrm{NH}, \mathrm{br} \mathrm{s}), 7.54(1 \mathrm{H}, \mathrm{d}, J=7.56 \mathrm{~Hz}), 7.21(1 \mathrm{H}, \mathrm{t}, J=2.8 \mathrm{~Hz}), 7.11-7.02(2 \mathrm{H}$, $\mathrm{m}), 6.58(1 \mathrm{H}, \mathrm{dd}, J=3.2,2.1 \mathrm{~Hz}), 5.45(1 \mathrm{H}, \mathrm{m}), 3.61(2 \mathrm{H}, \mathrm{d}, J=7.0 \mathrm{~Hz}), 1.85(3 \mathrm{H}, \mathrm{s}), 1.82$ $(3 \mathrm{H}, \mathrm{d}, J=1.2 \mathrm{~Hz}) ;{ }^{13} \mathrm{C}$ NMR $\left(75 \mathrm{MHz}, \mathrm{CDCl}_{3}\right) \delta 135.4,133.6,128.1,124.2,124.1,122.5$, 121.8, 120.3, 119.0, 103.2, 31.1, 26.0, 18.2; HRMS Calcd. for $\mathrm{C}_{13} \mathrm{H}_{15} \mathrm{NH}^{+}(\mathrm{M}+\mathrm{H})^{+}$186.1278, found 186.1282. Anal. Calcd. for $\mathrm{C}_{13} \mathrm{H}_{15} \mathrm{~N}$ : C, 84.28; H, 8.16; N, 7.56. Found: C, 84.28; H, $8.28 ; \mathrm{N}, 7.43$.

\section{7,7'-bis(3-methylbut-2-enyl)-2,3-dihydro-1' $H$-1,2'-biindole (5)}

A mixture of $3(0.56 \mathrm{~g}, 3.0 \mathrm{mmol})$ and activated manganese(IV) oxide $(0.78 \mathrm{~g}, 9.0 \mathrm{mmol})$ in chloroform $(30 \mathrm{~mL})$ was heated at reflux. After $1.5 \mathrm{~h}$, additional activated manganese(IV) oxide $(0.78 \mathrm{~g}, 9.0 \mathrm{mmol})$ was added to the reaction mixture. The mixture was kept refluxing for another $1.5 \mathrm{~h}$, allowed to cool, and filtered through a short plug of silica gel. Concentration of the filtrate under reduced pressure was followed by column chromatography (ethyl acetate:hexane 1:30) to yield the title compound $(0.19 \mathrm{~g}, 35 \%)$ as a greenish-yellow oil. $\mathrm{R}_{\mathrm{f}}=$ 0.19 (ethyl acetate:hexane 1:10). IR (neat) 3419, 2967, 2912, 2853, 1589, 1471, 1448, 1434, $1316,1100,1067,848,758,723 \mathrm{~cm}^{-1} ;{ }^{1} \mathrm{H}$ NMR $\left(300 \mathrm{MHz}, \mathrm{CDCl}_{3}\right) \delta 8.07(\mathrm{NH}$, br s), 7.20 $-7.16(2 \mathrm{H}, \mathrm{m}), 7.08(1 \mathrm{H}, \mathrm{d}, J=7.1 \mathrm{~Hz}), 6.94(1 \mathrm{H}, \mathrm{d}, J=7.6 \mathrm{~Hz}), 6.87-6.80(2 \mathrm{H}, \mathrm{m}), 6.43(1 \mathrm{H}$, $\mathrm{m}), 5.41(1 \mathrm{H}, \mathrm{m}), 5.14(1 \mathrm{H}, \mathrm{m}), 3.93(2 \mathrm{H}, \mathrm{t}, J=8.4 \mathrm{~Hz}), 3.54(2 \mathrm{H}, \mathrm{d}, J=6.9 \mathrm{~Hz}), 3.11(2 \mathrm{H}, \mathrm{t}$, $J=8.4 \mathrm{~Hz}), 2.83(2 \mathrm{H}, \mathrm{d}, J=7.1 \mathrm{~Hz}), 1.82(3 \mathrm{H}, \mathrm{s}), 1.78(3 \mathrm{H}, \mathrm{s}), 1.64(3 \mathrm{H}, \mathrm{s}), 1.38(3 \mathrm{H}, \mathrm{s}) ;{ }^{13} \mathrm{C}$ $\operatorname{NMR}\left(75 \mathrm{MHz}, \mathrm{CDCl}_{3}\right) \delta 148.5,143.1,133.6,133.0,132.6,132.4,128.5,128.3,126.8,124.7$, 124.5, 123.0, 122.6, 122.4, 120.6, 119.1,113.0, 103.1, 59.5,31.2, 30.7, 29.8, 26.02, 26.00, 18.3, 17.9; HRMS Calcd. for $\mathrm{C}_{26} \mathrm{H}_{30} \mathrm{~N}_{2} \mathrm{H}^{+}(\mathrm{M}+\mathrm{H})^{+} 371.2483$, found 371.2499 .

\section{Supplementary Material}

Refer to Web version on PubMed Central for supplementary material.

\section{Acknowledgements}

Financial support from the NIH (DK-60532) is gratefully acknowledged. We thank CEM Corp. for providing the microwave reactor. The assistance of C. Crotts in administrative support of this work is greatly appreciated.

\section{References}

1. Achenbach H, Renner C. Heterocycles 1985;23:2075-81.Achenbach H. Pure Appl Chem 1986;58:653-662.

2. Arai K, Sato S, Shimizu S, Nitta K, Yamamoto Y. Chem Pharm Bull 1981;29:1510-17.

3. Shimizu S, Yamamoto Y, Koshimura S. Chem Pharm Bull 1982;30:1896-9. [PubMed: 7116522]

4. a Benesova V, Samek Z, Herout V, Sorm F. Coll Czech Chem Commun 1969;34:1807-9. b Benesova V, Herout V, Sorm F. Coll Czech Chem Commun 1969;34:1810-14. c Delle Monache F, Delle Monache G, De Moraes e Souza MA, Cavalcanti MS, Chiappeta A. Gazz Chim Ital 1989;119:435-9. 
d Nagashima F, Sari Y, Tori M, Asakawa Y, Huneck S. Phytochemistry 1993;34:1341-3. e Agnaniet H, Menut C, Bessiere J-MJ. Essential Oil-Bearing Plants 2004;7:201-209. f Boti JB, Koukoua G, N'Guessan T, Yao Muselli A, Bernardini A-F, Casanova. J Phytochem Anal 2005;16:357-363.

5. a Pirrung MC, Li Z, Park K, Zhu J. J Org Chem 2002;67:7919-7926. [PubMed: 12423119] b Pirrung MC, Deng L, Li Z, Park K. J Org Chem 2002;67:8374-8388. [PubMed: 12444614] c Pirrung MC, Liu Y, Deng L, Halstead DK, Li Z, May JF, Wedel M, Austin DA, Webster NJG. J Am Chem Soc 2005;127:4609-4624. [PubMed: 15796526]

6. a Liu K, Wood HB, Jones AB. Tetrahedron Lett 1999;40:5119-5122. b Tatsuta K, Mukai H, Mitsumoto K. J Antibiot 2001;54:105-108. [PubMed: 11269708]

7. a Achenbach H, Franke D. Arch Pharm 1987;320:91-2. b Achenbach H, Franke D. Arch Pharm 1987;320:574-6.

8. Jing H, Shimada A, Maeda A, Arai Y, Goto M, Aoyagi Y, Ohta A. Chem Pharm Bull 1994;42:2779.

9. a Somei M, Funamoto T, Ota T. Heterocycles 1987;26:1783-4. b Muratake H, Natsume M. Heterocycles 1989;29:771-82. c Kondo Y, Kojima S, Sakamoto T. Heterocycles 1996;43:2741-2746. d Hartung CG, Fecher A, Chapell B, Snieckus V. Org Lett 2003;5:1899-1902. [PubMed: 12762681]

10. Pirrung MC, Wedel M, Zhao Y. Synlett 2002:143-145.

11. a Hennion GF, Hanzel RS. J Am Chem Soc 1960;82:4908-12. b Easton NR, Dillard RD, Doran WJ, Livezey M, Morrison DE. J Org Chem 1961;26:3772-6. c Barmettler P, Hansen H. J Helv Chim Acta 1990;73:1515-73. d Wipf P, Venkatraman S. J Org Chem 1996;61:6517-6522. [PubMed: 11667514]

12. a Roe JM, Webster RAB, Ganesan A. Org Lett 2003;5:2825-2827. [PubMed: 12889884] b Izumi T, Kasahara A. J Heterocyclic Chem 1990;27:1173-5.

13. Cooper MA, Lucas MA, Taylor JM, Ward AD, Williamson NM. Synthesis 2001:621-625.

14. Walsh DA, Moran HW, Shamblee DA, Uwaydah IM, Welstead WJ, Sancilio LF, Dannenburg WN. J Med Chem 1984;27:1379-1388. [PubMed: 6436487]

15. Stachel SJ, Habeeb RL, Van Vranken DL. J Am Chem Soc 1996;118:1225-1226.

16. a Sugiyama H, Yokokawa F, Aoyama T, Shioiri T. Tetrahedron Lett 2001;42:7277-7280. b Hansen DB, Lewis AS, Gavalas SJ, Joullie MM. Tetrahedron: Asymmetry 2006;17:15-21. c Yokokawa F, Sugiyama H, Aoyama T, Shioiri T. Synthesis 2004:1476-1480. 
<smiles>c1ccc2c(c1)CCN2</smiles>

indoline

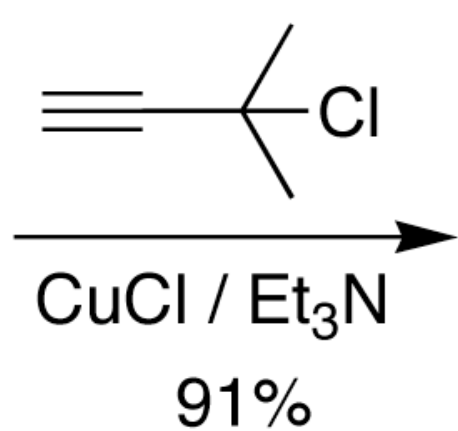

$91 \%$

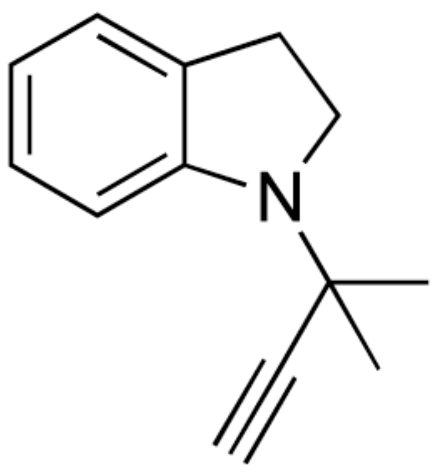

1

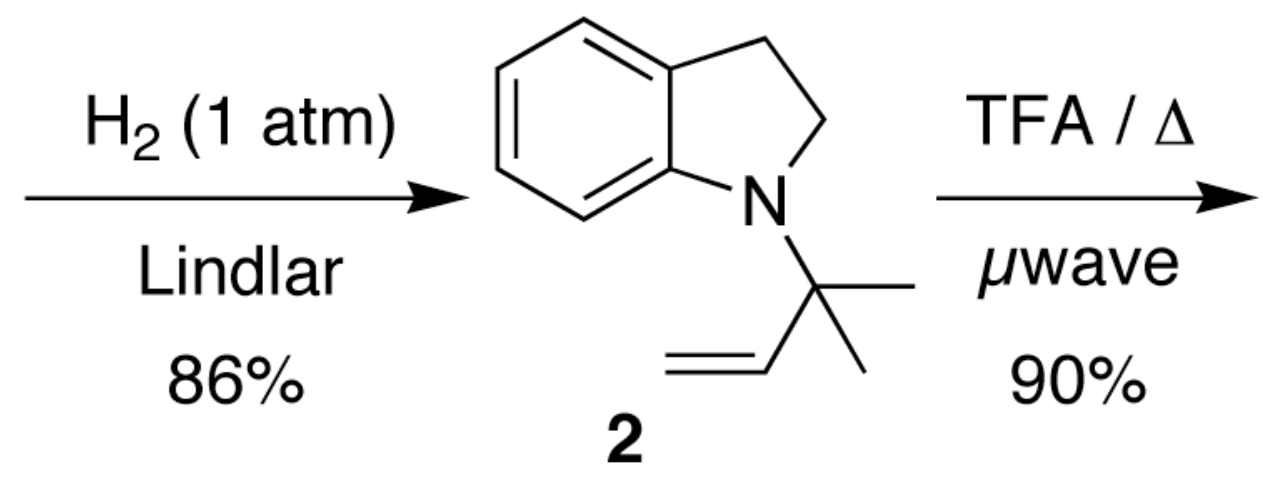

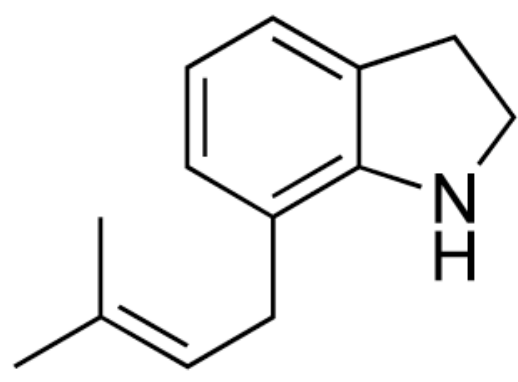

3

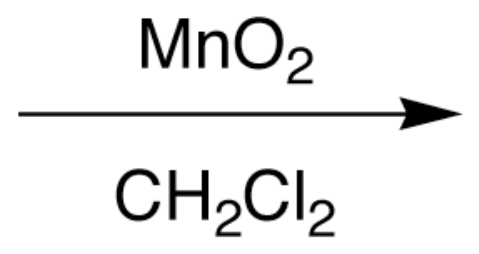

$88 \%$

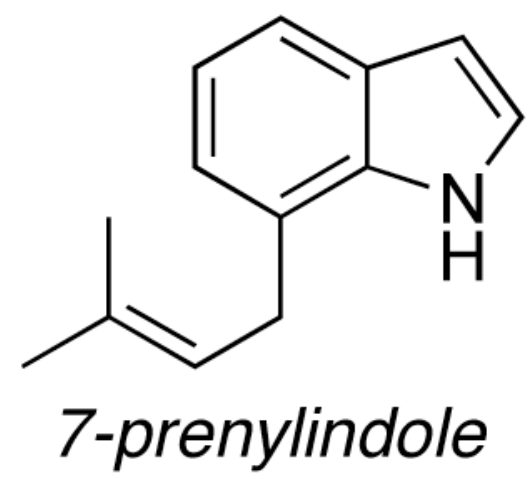

Scheme 1.

The preparation of 7-prenylindole 


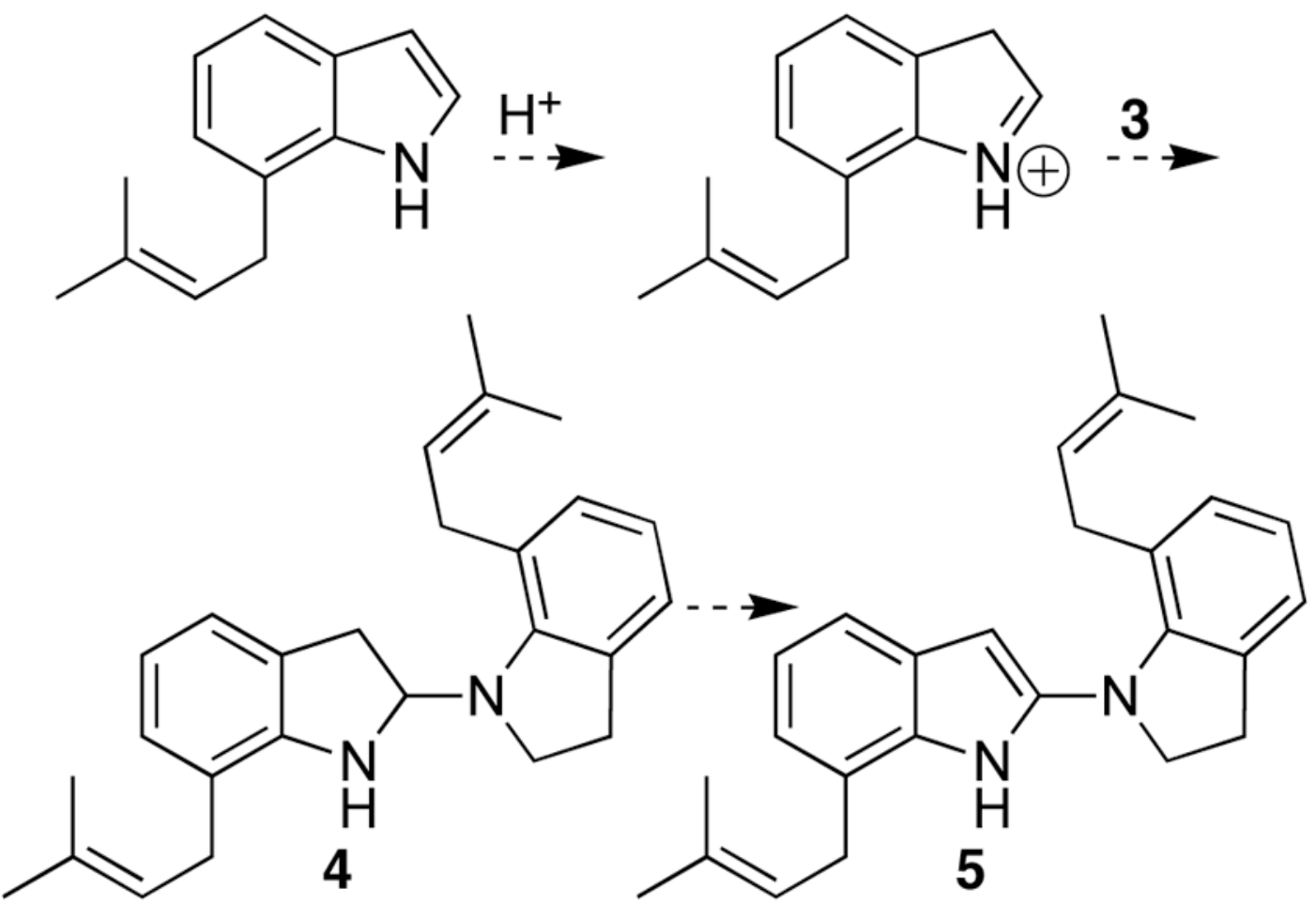

Scheme 2.

The formation of a 7-prenylindole/7-prenylindoline dimer 

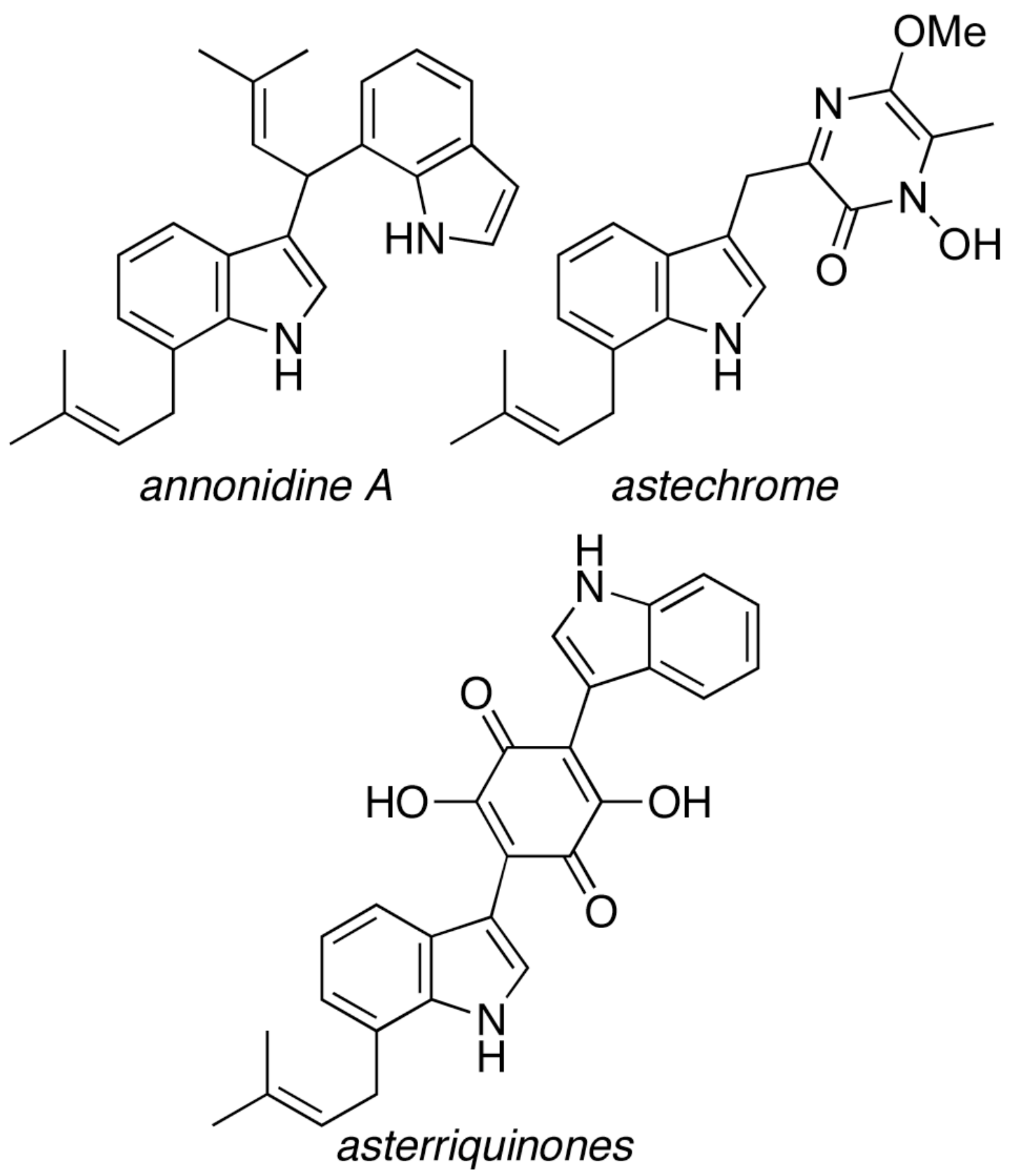

Chart 1.

7-Prenylindole-containing natural products 